\title{
Lenguaje y acción: creencias, instituciones y política ${ }^{1}$
}

\section{Language and action: beliefs, institutions and politics}

\section{FREDDY SANTAMARÍA VELASCO}

Doctor en Filosofía y Letras Universidad Pontificia Bolivariana freddy.santamariave@upb.edu.co

\section{SIMÓN RUIZ-MARTÍNEZ}

Candidato a Doctor en Estudios Políticos y Jurídicos

Universidad Pontificia Bolivariana simon.ruizm@upb.edu.co
Cómo citar este artículo en APA: Santamaria, F. y RuizMartínez, S. (2021).

Lenguaje y acción: creencias, instituciones y política. Analecta Política, 11(20), 86-108. doi: https://doi. org/10.18566/apolit. v11n20.a05

Fecha de recepción: 27.03.2021 Fecha de aceptación: 24.05.2021

\section{Resumen}

Este artículo busca consolidar las bases conceptuales en tanto herramientas analíticas de una investigación social basada en concepciones fuertes de lenguaje y acción. Ello es realizado a partir del esclarecimiento de qué se puede entender como lenguaje y cómo esto deriva necesariamente en la acción humana. Tres componentes fundamentales se desarrollan para la investigación social en este sentido: creencias, discurso y comportamiento. Cada uno de ellos se desarrolla de manera particular, con ejemplificaciones sobre la manera en que pueden

Este artículo está inscrito en el proyecto de investigación "Prácticas discursivas y la construcción colectiva del concepto de Seguridad desde el territorio (Medellín)"; radicado CIDI: 565C-03/20-36 del Grupo de Investigación en Estudios Políticos de la Universidad Pontificia Bolivariana 
ser llevados a cabo. El aglutinante de toda la propuesta es la noción práctica de razón, que no solo particulariza la concepción del lenguaje presupuesta, sino que también clarifica el alcance de las preguntas investigativas que vale la pena indagar en el ámbito de las ciencias sociales.

Palabras clave: ciencias sociales; filosofía de la acción; pragmática; análisis del discurso; teoría de la elección racional

\section{Abstract}

This article seeks to consolidate the conceptual bases as analytical tools of a social research based on strong conceptions of language and action. This is carried out from the clarification of what can be understood as language and how this necessarily results in human action. Three fundamental components are developed for social research in this regard: beliefs, discourse, and behavior. Each of them is developed in a particular way, with examples of how they can be performed. The binding element of the whole proposal is the practical notion of reason, which not only specifies the conception of language presupposed, but also clarifies the scope of the research questions that are worth investigating in the field of social sciences.

Keywords: social sciences; philosophy of action; pragmatics; discourse analysis; rational choice theory. 


\section{El problema del lenguaje}

Los problemas de investigación en ciencias sociales se enfrentan, en la mayoría de los casos, a una doble complejidad: a) ¡cómo es posible abordar de manera rigurosa problemas que son suscitados mayoritariamente por la ambigüedad del lenguaje ordinario? y b) ¿cómo hacer un estudio científico adecuado que de hecho esté referido a la aplicación práctica de estas investigaciones (esto es, a la transformación social)? La primera pregunta apunta a la compleja discusión de nociones como 'valor', 'poder', 'sociedad', e incluso 'política'. ¿Es siempre claro lo que entendemos por ellos? El valor del economista no es el mismo del sociólogo, y la sociedad del historiador no es la misma del politólogo. ${ }^{2}$ Esta ambigüedad parte de la necesidad del investigador social de hablar de lo que un individuo hace. Y las acciones humanas son tantas y tan variadas como apuestas metodológicas. La segunda pregunta toca una cuestión mucho más específica del ejercicio investigativo: ‘tiene sentido una investigación social desligada de su aplicabilidad práctica? Pero, de manera más compleja, ¿cuál sería la manera de relacionar la teoría social con su aplicabilidad? ¿Es prioritaria la aplicación y a ella se subordina la teoría? ¿O deben formularse modelos y usarse o descartarse de acuerdo con su éxito aplicativo?

Esta complejidad dual hecha explícita por Sartori (2002) motiva la definición de un enfoque de investigación centrado en las prácticas lingüísticas que definen el ámbito de acción humana cuyo núcleo central resuelva, o, mejor aún, disuelva, el problema en cuestión. Lenguaje y acción ${ }^{3}$, pensado como un enfoque para la investigación en ciencias sociales, busca, entonces, hacer espuria la pregunta: ¿cómo es posible investigar a la sociedad ad intra, ${ }^{4}$ desde su leguaje ordinario y sus prácticas cotidianas?

Cabe precisar que la discusión que se presentará a continuación no se concentra en la justificación del carácter ad intra de la investigación social. Se supone que la neutralidad u objetividad pretendida por el investigador se ciña al alcance de una comunidad lingüística o, de manera simple, que es posible la objetividad en investigaciones sociales (Santamaría y Ruiz-Martínez, 2019; Ruiz-Martínez y Rosanía Maza, 2021).

Debería decirse, no es necesariamente la misma.

3 A partir de este momento, se usará la mayúscula inicial junto con las cursivas para designar el enfoque de investigación.

4 A diferencia de las ciencias naturales que pueden lograr un punto arquimídeo sobre el cual basar sus experimentos, es imposible, para el investigador social, encontrar un punto por fuera de las prácticas sociales. Este es el carácter interior al que se alude. 
De lo que se trata, entonces, es de presentar el alcance del lenguaje y la acción y su relación en la comprensión y explicación de todo suceso o hecho social. Ahora bien, cuando se asume que todo suceso o hecho social puede ser abordado desde tal enfoque, no se trata de forzar la descripción a lo que otros enfoques entienden como hechos, sucesos o fenómenos sociales, sino, por el contrario, de "romper la costra muerta de la tradición y la convención, romper los grilletes que nos encadenan a preconcepciones heredadas, para así alcanzar una nueva forma de ver las cosas"s (Waismann, 1968, p. 32). La primera y más importante definición de Lenguaje y acción es, pues, una visión, esto es, una visión de la acción social desde la pragmática lingüística. La pragmática resalta, desde la teoría del signo presentada magistralmente por Peirce (2012) en el siglo XIX, la fuerza performativa del lenguaje. Según Korta y Perry (2020) esta disciplina,

se ocupa de los enunciados, con lo que nos referiremos a eventos específicos, los actos intencionales de los hablantes en momentos y lugares, que en general, involucran el lenguaje. [...]. La pragmática a veces se caracteriza por tratar los efectos del contexto. Esto es equivalente a decir que se trata de enunciados, si uno se refiere colectivamente a todos los hechos que pueden variar de un enunciado a otro como "contexto". (Korta y Perry, 2020)

Esta visión desde la pragmática la componen diversos aspectos clave: creencias, discurso y comportamiento, que no son una selección arbitraria de conceptos, sino que están profundamente hilvanados por la noción pragmática de razón, de racionalidad. Distinguimos la pragmática como escuela filosófica, frecuentemente denominada pragmatismo o pragmaticismo, del enfoque de Lenguaje y acción para evitar equívocos: no se trata de una lectura filosófica de los problemas de ciencias sociales, sino más bien de adecuar las herramientas investigativas a las prácticas efectivas, es decir, lo que de hecho hacemos cotidianamente. No obstante, la pragmática social es el fundamento conceptual de este enfoque investigativo (Brandom, 2008; Peirce, 2012; Searle, 2010; Wittgenstein, 1969, 2009). Por esta razón, partiremos de una concepción generalizada del lenguaje desde el pragmatismo para preparar el suelo sobre el cual pueda erigirse el campamento científico. ${ }^{6}$

5 Las traducciones son nuestras.

6 El científico social no debe ser un arquitecto; no se trata de construir edificios, sino, como el buen arqueólogo, de encontrar evidencias y describir posibles patrones racionales de comportamiento. El campamento arqueológico del saber social debe, entonces, partir de la realización de que su labor es eminentemente activa y vinculada con el trabajo de campo (Wittgenstein, 2009, §217). 


\section{Lenguaje y pragmática}

Dos ideas componen, de manera simple, la definición pragmática del lenguaje. La primera cuestiona la concepción moderna del lenguaje afirmando que las palabras son también actos (Witttgenstein, 2009, \$546). La segunda hace explícita una simple conclusión práctica: dominar un lenguaje es dominar una técnica (Wittgenstein, 2009, \$199). Antes de abordar a fondo estas dos ideas, es importante describir de manera somera el vuelco que hace el pragmatismo de la concepción moderna del lenguaje. Para dicha concepción, aún sostenida tanto por académicos como por personas en su cotidianidad, el lenguaje es un código para la comunicación del pensamiento (Acero, 2007, pp. 12 y ss.; Locke, 1999, pp. 391 y ss.). Así, el lenguaje sirve, según quienes mantienen todavía este enfoque, para vehicular conceptos (en la academia) y para expresar sentimientos, emociones y opiniones (en la cotidianidad). Este exorcismo lingüístico, que extrae fenómenos mentales de palabras y conceptos, no tiene cabida en la posición pragmatista, según la cual usamos el lenguaje para describir las acciones que realizamos, para evaluarlas, para normativizarlas; usamos el lenguaje para evidenciar en el mundo de la acción nuestros estados mentales. En este sentido, el lenguaje termina por ser conducta.

La pragmática no es otra cosa que el reconocimiento de la función del lenguaje, es decir, como medio de intercomunicación social, consistente básicamente en decir algo a alguien, y que ese "algo" sea significativo para quien lo escucha. Esto quiere decir que el significado lo da el uso de tal o cual enunciado dentro de un entramado social. Un uso del lenguaje, en cualquier caso, preponderantemente práctico. Poseer lenguaje es lo que propiamente nos hace sujetos sociales. El lenguaje remite al hombre social, no al hombre individual. No hay lenguaje sin hombre social ni hombre social sin lenguaje. Las personas somos seres sociales, pero en virtud de que somos seres lingüísticos. Nos relacionamos como sujetos hablantes; entre el lenguaje y el mundo, pues es desde esta relación como se materializa propiamente el análisis de la llamada "realidad". De ahí que el papel que desempeñan nuestras prácticas será hacer explícito lo implícito, porque en el lenguaje encontramos lo que nosotros mismos hemos introducido en él mediante nuestras prácticas sociales por ser "miembros de una comunidad" (Santamaría y Ruiz Martínez, 2019); acción y explicitación. Este encuentro, entre la acción misma y el hacer, explicita tales prácticas: no son simplemente palabras, con ellas hacemos mundos, pues nos definimos "como criaturas gobernadas por normas, en contraste con criaturas que siguen reglas puramente naturales, por actitudes normativas que mostramos, unas actitudes con las cuales expresamos que comprendemos o concebimos en la práctica nuestra conducta como gobernada por normas" (Brandom, 2005, p. 79). Las prácticas discursivas, como afirma Bran- 
dom, incorporan cosas reales, a saber: el arte, la religión, el amor, la amistad, la educación y la política, es decir, la cultura.

Desde una concepción pragmática, el lenguaje no es más que otra de las actividades que realizamos normalmente. Es importante clarificar: no se trata de una sustitución de una cosa por otra, de fenómenos mentales a actos lingüísticos como eventos, como, en cierta medida, pretende Davidson (2001, p. 15). Seguir esta vía argumentativa sería enredar aún más una elucubración filosófica inane. Hacer explícitos los hábitos de comportamiento quiere decir que el lenguaje se ubica en el mismo plano que las acciones; que el lenguaje es, en el comienzo, una acción (Wittgenstein, 1969, \$402).

Así, se arriba a la primera idea expuesta al comienzo de esta sección: las palabras son también actos. Debería decirse, las palabras son también compromisos. ${ }^{7}$ Hacer explícito un hábito, una acción, quiere decir dar razones de su ejecución (Brandom, 2005). No se necesita demasiada reflexión para reconocer que en la mayoría de nuestras acciones no hacemos uso de expresiones lingüísticas. Solo cuando se pregunta por las razones de una acción es que tiene sentido el uso del lengua$j e$. De esto se trata ser racional, racionalizar una acción: dar o pedir las razones por una conducta. Decir de tal o cual persona que es racional quiere decir, entonces, que, o bien se encuentran razones para que actúe como lo hace, o bien que puede preguntársele: “¿Por qué hiciste A cuando debiste hacer B?”, es decir, “¿Por qué no seguiste la regla que define la pertenencia a nuestra comunidad?”. Por eso, las palabras nos comprometen con la consecuencia de nuestros actos.

Centrar la atención en la consecución de una acción lleva a la segunda idea: dominar un lenguaje es dominar una técnica. De manera simple, la técnica que dominamos es la capacidad de dar y pedir razones sobre nuestros actos y aquellos llevados a cabo por los demás. No es fortuito, entonces, que la competencia lingüistica esté fuertemente relacionada con la racionalidad, esto es, la capacidad de racionalizar nuestras acciones, de dar y pedir razones de nuestro comportamiento (Brandom, 2005; Sellars, 1997).

Quizá la palabra 'compromiso' pueda llevar a equívocos. Cuando se define el lenguaje como una técnica, como una práctica de dar y pedir razones, no implica que siempre tengamos que ir por el mundo narrando nuestras propias acciones,

7 La expresión original es "Worte sind auch Taten". El término en alemán puede traducirse plenamente por acción. Pero la articulación que esta idea tiene con el seguimiento de reglas (infra) permite substituir el término por comprometerse. 
expresando las razones que tenemos para hacer lo que hacemos. El dominio de una técnica no requiere justificaciones verbales. Lo que sí requiere justificación es el fracaso en dicha técnica, es decir, el incumplimiento del compromiso práctico que articula una acción a la evaluación racional de pertenencia a esa comunidad. La idea de dominio del seguimiento de reglas alude, entonces, a la idea de competencia. Es a partir del fracaso en nuestra competencia lingüística que surge la necesidad de dar y pedir razones. Por esta razón, las palabras no son simpliciter, sino son también compromiso.

La composición de las dos ideas pragmáticas da lugar a la profunda y simple noción de seguimiento de regla, piedra angular de la visión pragmática del lenguaje. De dicha noción nos ocuparemos en este momento.

\section{La práctica: seguir reglas}

La noción de seguimiento de regla es tan simple que una vez que se formula se presta para un sinnúmero de malentendidos: seguir una regla es una práctica (Wittgenstein, 2009, \$202). Eso es todo. Esperar algo más del lenguaje, del comportamiento humano, de la acción intencional, es atribuirle cosas que no van al caso. Seguir reglas es, en definitiva, algo que ya hacemos. Por eso, una de las mejores descripciones de seguimiento de reglas raya en la trivialidad:

Cuanto más reflexiono sobre las reglas, más pienso que Wittgenstein estaba en lo cierto cuando encontraba algo inefable en una situación lingüística; algo que puede ser compartido, pero no comunicado. Vimos que para que algo sea efectivamente una regla debe vivir en el comportamiento, comportamiento adecuado a reglas [rule-regulated behavior], incluso comportamiento contrario a una regla [rule-violating behavior]. En nuestra práctica lingüística, siempre actuamos dentro de un marco de reglas vivas. Hablar acerca de reglas es salirse del hablar-sobre reglas hacia otro marco de reglas vivas. (Sellars, 2005, p.134)

Para Wittgenstein (2009), quien desarrolla la noción de seguimiento de reglas y juegos de lenguaje, todo juego supone una sociedad, una forma de vida (\$\$199 y ss.). ${ }^{8}$ De la misma forma, si este tipo de arte, con sus nuevas reglas de juego, es

8 Rodríguez Ortiz y Ramírez-Vallejo (2021) muestran la relación entre los "juegos del lenguaje" y los "actos de habla" expuestos inicialmente por Austin y reelaborados por Searle, por ejemplo, en "la propuesta searleana, las acciones que los juegos del lenguaje convocan se convierten 
"asumido" por una comunidad lingüística, su significatividad está asegurada, no depende de la aceptación o no de un miembro particular.

Dicho carácter social del juego implica una idea crucial para el propósito del investigador social: no se siguen las reglas privadamente, pues, cuando aprendemos un lenguaje no solo aprendemos una técnica, sino todo un complejo conjunto de técnicas en relación con otros. Hablar un lenguaje no es tan solo participar en una práctica, sino también en muy distintas prácticas. Se podría decir que un lenguaje es una práctica compleja compuesta de un número de prácticas (Fann, 2015), esto es, solo es posible seguir una regla siendo miembro de una comunidad lingüistica que de igual modo siga reglas.

Preguntas como “¿dónde está la regla?”, “¿cómo sabemos que es la regla correcta?" y "¿es posible equivocarse siguiendo esta o esa regla?" pierden de vista el punto central: la regla es un comportamiento esperado que se hace o no explícito. Es crucial dejar abierta la posibilidad de no hacer explícita la regla, de fallar. Porque es allí donde se evidencia el sentido realmente práctico del seguimiento de reglas: no se trata de describir determinaciones causales del comportamiento humano, sino de encontrar que esperan los agentes racionales de sus semejantes en contextos particulares, o lo que Sartori (2002, p. 59) denomina "indeterminaciones causales".

Para entender un poco esta noción, que se denomina adiestramiento siguiendo a McDowell (1998) y Santamaría y Ruiz Martínez (2019), piénsese en la adquisición de la competencia en una práctica: no venimos al mundo como filósofos expertos en la deontología kantiana o en el derecho harteano, sino que actuamos de acuerdo con lo que hemos aprendido desde pequeños. Nos formamos en unas prácticas específicas en las que somos adiestrados: hablar español, saludar cuando alguien nos extiende la mano, sentarnos en una silla, contar con los dedos, mirar la hora en un reloj, etc. Esto quiere decir que no se trata de entender si la regla es algo en el mundo, si es una cosa, un evento, etc., sino de entender que "los niños no aprenden que existen libros, que existen sillas, etc.; aprenden a traer libros, a sentarse en sillas,

en la génesis de la realidad social. En palabras de Searle, su filosofía de actos de habla, y con esta, los estudios sobre la construcción de la realidad social, se consideran "teorías institucionales de la comunicación". El paso discursivo de Wittgenstein del estudio lógico del lenguaje al estudio de los lenguajes comunes y las acciones que estos provocan es algo que también está vigente desde el inicio de la obra searleana, una relación esencial que -como él mismo lo acepta en la parte final del capítulo IV de Speech Acts - está presente en las teorías de Austin, de Wittgenstein y en la suya propia". 


\section{Freddy Santamaría Velasco, Simón Ruiz-Martínez \\ Lenguaje y acción: creencias, instituciones y política}

etc." (Wittgenstein, 1969, \$476). Ciertamente, las prácticas lingüísticas que analiza el científico social no surgen de un proceso de raciocinio $(\$ 475)$.

En este sentido, somos sociales, por supuesto, pero también individuos egoístas. Nuestras razones, particulares creencias y elecciones gobiernan nuestras preferencias y deseos, así es como para Schick (2000) el deseo y la creencia pueden ser considerados motivos o bases de la acción, pero una razón completa, una explicación causal de la acción, requiere, además, que el agente interprete la acción "como algo que quiere que sea hecho". Una aproximación epistemológica a la relación entre el lenguaje y la acción comprende la necesidad de responder cuáles son los principios y modelos racionalmente justificados, para establecer la normatividad sobre la estática y dinámica de nuestros sistemas de creencias y conocimiento. Este sería el fundamento para establecer las relaciones de dichos fundamentos epistémicos con la agencia racional individual y colectiva que se hace patente en el lenguaje.

¿De qué se trata entonces el seguimiento de reglas? Se trata de comprender $a$ qué comunidad se pertenece, qué juego se juega, qué cabe esperar de alguien más de manera egoísta (Santamaría y Ruiz-Martínez, 2019). Seguir una regla siempre es una cuestión pública. Si se quiere, el punto de partida de cualquier sentido o noción de lo público. Se trata de comprender qué cuenta como racional para aquellos que juegan el mismo juego que nosotros hemos aprendido. Es decir, de discriminar las razones que tiene sentido dar y pedir en un contexto social particular.

\section{Formalización de la institución}

El espacio público, el sentido de la interacción social de diferentes agentes, se hace inteligible a partir del seguimiento de reglas. Una de las condiciones básicas para un liberalismo político adecuado es, entre otras cosas, que yazgan implícitas las normas sociales en las prácticas explícitas de una comunidad política particular (Rawls, 1996, pp. 13 y ss.). Analizada con un poco de detenimiento, la anterior afirmación implica un pluralismo práctico bastante importante en el quehacer de la ciencia social. No es posible discriminar razones por fuera de sistemas de reglas específicos. Es Rawls (1955) mismo quien comienza a caracterizar que una adecuada concepción de la racionalidad debe separar las reglas particulares del sistema de reglas completo que define la institución. 
Pero ello implica una contextualización especial, tal y como es desarrollada por Williams (2008): la normatividad del comportamiento humano no es generalizada: si bien somos animales normativos, las normas que seguimos no son homogéneas. Cada concepción política, cada justificación del sistema de reglas que regula nuestras interacciones políticas, debe ser justificado de acuerdo con condiciones de legitimidad que tengan sentido práctico dentro del contexto histórico y social de dicha sociedad. Confróntese esto, por ejemplo, con las condiciones de legitimidad que Williams le atribuye al liberalismo contemporáneo:

Los defensores del liberalismo van, en primer lugar, a elevar el estándar de quienes son reconocidos como desaventajados. Esto, debido a que elevan sus expectativas sobre lo que el Estado puede hacer; además, adoptan, quizá, porque están en una posición apta para ello, estándares más exigentes sobre lo que cuenta como una amenaza a los intereses vitales de las personas, amenazas entendidas en términos del primer problema. (p. 7)

El primer problema es el aseguramiento de las condiciones que se asumen como básicas; algo que este autor denomina la demanda básica de legitimidad. En este sentido, qué cuenta como básico es un asunto práctico de definición de reglas constitutivas de la comunidad democrática y liberal en cuestión. Por eso, sostiene Williams (2008), "reconocerán, por ejemplo, el derecho a la libre expresión; en primer lugar, porque es importante que los ciudadanos sepan si la demanda básica de legitimidad está siendo cumplida por parte del Estado" (p. 8). Para un investigador social, la contextualización de la racionalidad de las instituciones políticas debe darse en el marco de una discriminación práctica de lo que los jugadores de tal comunidad esperan de la institución que los cobija.

Que las reglas que definen una comunidad no sean homogéneas en la totalidad de la especie humana no debe ser algo sorprendente. Si tiene un lugar en la argumentación en curso, es solo para introducir la cuestión de la normatividad. Pero no una deontológica y abstracta, sino una práctica, en la que en el principio es la acción (Wittgenstein, 1969, \$402). Nótese, por ejemplo, la cercanía de este enfoque con la defensa del republicanismo de Pettit (1997):

Supóngase que hay normas establecidas, tanto a lo largo de una sociedad como al interior de los grupos relevantes que la componen, que apoyan cierto tipo de leyes republicanas. En este caso, tanto las normas como las leyes tendrán un carácter republicano: serán consistentes con, y de hecho un apoyo importante, una administración de bienes en la que todos disfruten de libertad como no dominación [...]. Cuando dije que las leyes republicanas deben estar contenidas en una red de 
normas civiles, pude haber dicho también que las leyes republicanas deben estar soportadas por hábitos de virtudes civiles o de buena ciudadanía. (p. 245)

La constitución de una república es, de acuerdo con lo anterior, indisociable de una normatividad fundada en la compatibilidad del comportamiento con las leyes que definen un Estado jurídico institucional. Leído en orden inverso, solo a partir de las normas comunitarias tiene sentido la constitución de una institución política efectiva. En esta medida, es fundamental para la investigación social tener a la mano herramientas que pongan sobre la mesa la compatibilidad entre las razones para la acción de un actor institucional y las normas sociales que rigen a esta comunidad.

Además, Pettit (1997) llega a afirmar que toda acción social es la composición de acciones de agentes individuales, aun cuando la composición pueda superar explicativamente las acciones individuales. De este modo, solo se puede entender al sujeto desde la pertenencia a una comunidad y donde tienen importancia los principios políticos, las reglas asumidas y la construcción de instituciones públicas. Para Pettit, ninguna acción tiene sentido en abstracto, sino en el ámbito social, de ahí que entiende la filosofía política, por ende, social, como :

- Anticolectivista. En contra de toda concepción según la cual los individuos son meros juguetes de fuerzas sociales agregadas, meros números en un juego de azar histórico, peones en marcha hacia un destino histórico.

- Antiatomista. En la que se critica la idea de individuo solitario en la sociedad, pues, como advierte Pettit (1997), "la noción de individuo solitario es ilusoria: las personas dependen unas de otras, a través de más de un nexo causal, incluso para su misma capacidad de pensar; son, esencialmente, criaturas sociales" (VII).

Una conciencia colectiva (coawareness) derivada de las conciencias personales y que permiten entender el estado de cosas relativo a las interacciones sociales. ${ }^{9}$ Esta precisión es crucial en la medida en que la complementariedad entre la in-

9 Pettit parte de la tradicion wittgensteiniana y de la idea de comunidad peirciana. Entiende que toda práctica lingüística supone una comunidad de trasfondo, una forma de vida (Lebensform), unos hábitos que se realiza dentro de cierta práctica de vida. De este modo, toda forma de vida, con sus nuevas reglas de juego, es asumida por una comunidad lingüística y su significatividad está asegurada. Aprendemos un lenguaje, pero no solo una técnica, sino todo un complejo conjunto de técnicas. Hablar un lenguaje no es tan solo participar en una práctica, sino en muy distintas, es decir, solo es posible seguir una regla siendo miembro de una comunidad lingüística que de igual modo siga reglas (Brandom, 2005; Santamaría y RuizMartínez, 2019). 
tención individual y colectiva que daría sentido a la institución política relaciona la práctica de dar y pedir razones entre individuos con aquella que se da entre actores individuales y actores colectivos o representativos, entre los cuales, el Estado es, sin duda, el más sobresaliente.

Para dilucidar estas herramientas desde el enfoque de Lenguaje y acción, la idea que se defenderá será, de acuerdo con lo anterior, que es a partir de razones que se erigen las instituciones sociales. La primera y más importante clarificación se desarrolla a partir de Tomasini (2016, p. 18), para quien una razón es un tipo de explicación que hace inteligible el comportamiento humano. Esta definición se elabora a partir de la diferenciación que tiene una razón para actuar con la causa de una acción. Si bien es cierto que la causa de una acción puede tener un sentido dentro de un contexto científico específico (esto es, la causa neurológica de un determinado comportamiento para un neurólogo, etc.), en un sentido lato, el comportamiento social no es descriptible desde el punto de vista de las causas, sino que es definido a partir de razones; es decir, encontrar un detonador causal no es lo mismo que hacer una acción comprensible. ${ }^{10}$

Esta diferenciación, ciertamente articulada con la de seguimiento de reglas (Tomasini, 2016, p. 29), se decanta a partir del análisis de la pregunta “¿por qué?” Ahora bien, ¿cómo se pasa de este por qué de la inteligibilidad y justificación de la acción a la institución social? Para zanjar esta cuestión, primero, hay que definir qué se entiende por institución social: un sistema de reglas coherente para la coordinación de la interacción social. Esta definición generalizada es coherente con las propuestas más tradicionales de Rawls $(1955,1999)$ y Searle $(1995,2010)$. Es decir, el sistema de reglas que define la institución social dota de sentido la estructura básica de las prácticas políticas (Rawls, 1999) y permite la discriminación de hechos institucionales objetivos a partir de los cuales puede avanzarse en mejores concepciones de valores sociales (Searle, 2010). Cabe anotar en este punto que, para Searle, el uso del lenguaje constituye las instituciones sociales y es a través de los actos de habla que se devela la intención colectiva de quienes conforman tal sociedad.

En este sentido, los actos de habla crean hechos institucionales. En otras palabras, el uso del lenguaje nos permite, como seres humanos, comprender los hechos brutos del mundo en el que habitamos y crear hechos institucionales en la sociedad a la cual pertenecemos; hechos institucionales en cuya naturaleza, como bien se ha resaltado, subyace el lenguaje. Sin lenguaje no sería posible hablar de

10 Elster (2015, pp. 3 y ss.) apunta a una idea similar, aunque no precisamente exacta, en su distinción entre hecho y suceso. 
una realidad social. Hacemos explícito lo implícito. De ahí que dominar la palabra sea dominar las acciones y quien consigue el dominio social de la palabra, es decir, el poder para determinar la relación entre lenguaje, acción e instituciones sociales, pueda llegar a naturalizar tal relación y dominar a todo un pueblo.

Como se trata de entender el alcance del enfoque de Lenguaje y acción, más profundizar en estos aspectos importantísimos, la argumentación se limitará a cerrar el espacio entre razones e instituciones, y así dejar el camino para abordar la cuestión de valor social en la sección siguiente. En este sentido, se trata de pasar de las preguntas sobre la inteligibilidad y justificación de una acción a la definición o implementación del sistema de reglas institucional (institucionalización social en breve).

Para ello, echaremos mano de la propuesta epistemológica de Sosa (2011), para quien el conocimiento tiene grados de plenitud, definidos a partir de tres niveles: la precisión (accuracy), la destreza (adroitness) y la aptitud (aptness). Si bien la aplicación de esta estructura se orienta a la discriminación de estados experienciales bajo los cuales pueda hablarse de conocimiento pleno de un agente (Sosa, 2011, pp. 108 y ss.), su base pragmática (en palabras de Sosa, performativa) la hace claramente compatible con el argumento desarrollado. Piénsese, entonces, que, para que la justificación y la inteligibilidad de una acción cuenten como plenamente institucionales, deben cumplir esos mismos tres criterios: precisas, diestras y aptas. ¿Pero qué quiere decir que una razón es precisa, diestra y apta?

Una razón es precisa cuando atañe al comportamiento evidenciado. La precisión hace explícito el contexto relevante en el cual se lleva a cabo una acción, contexto que involucra aspectos fisiológicos, sociológicos, históricos, etc. Si preguntamos por qué alguien está comiendo, una razón precisa es que tenía hambre. En un ejemplo más complejo, si preguntamos por qué un candidato formó una coalición con otro partido, una razón precisa es la adición de votos que ello representaría. Por otro lado, una razón imprecisa sería decir que lo hizo porque tenía hambre.

Hablamos de razones diestras cuando el comportamiento evidenciado responde a intenciones efectivas del agente y no a contingencias particulares. De manera simple, la intencionalidad es un estado particular que nos lleva a realizar una acción (Searle, 2010, pp. 25 y ss.). Es, si se quiere, la motivación para la realización de la acción. Si la precisión alude al contexto de la acción, la destreza alude a la individualidad de la acción. Si decimos que un gobierno redujo las tasas de homicidio durante su administración, la razón diestra requiere que esta reducción 
responda a las políticas realizadas por el Gobierno (a su intención como ente administrativo) y no a una tregua entre grupos armados.

Para definir una razón apta, basta con traer a colación la definición de aptitud que da el mismo Sosa (2011): "una acción [performance] es apta si su éxito manifiesta una competencia basada en el agente (en condiciones relevantemente apropiadas)" (p. 7). La aptitud es el resultado de una acción que es tanto precisa como diestra. Esto no quiere decir que la aptitud se reduzca a la destreza y a la precisión, sino que depende del agente llevar a cabo una acción en el contexto adecuado con la intención adecuada. La aptitud es la consecución adecuada de la instancia de la acción. Retomando el ejemplo anterior, es que un gobierno quiera disminuir la tasa de homicidios y, de hecho, lo haga por su propia cuenta, esto es, por el resultado de sus acciones.

Sin embargo, no basta con estos tres niveles para definir adecuadamente la institucionalización social. Para ello, hay que rescatar una última noción de la estructura de Sosa (2011): la meta-aptitud. En ella, importa la selección de las instancias específicas para la ejecución de una acción y no tanto su consecución. Puede decirse que es una discriminación estratégica de la competencia. El Gobierno puede fallar en su intento de disminución de la tasa de homicidios porque se apresuró en implementar la política, por ejemplo, no haciendo pedagogía suficiente sobre una aplicación de denuncios. La institucionalización del comportamiento alude, entonces, tanto al cumplimiento de las tres condiciones básicas como condiciones necesarias como al cumplimiento de la meta-aptitud como condición suficiente.

El análisis de las instituciones sociales queda así definido como un análisis estructurado de las diferentes interacciones según la precisión, destreza y aptitud de las razones de los actores relevantes, así como de la selección estratégica del momento para llevar a cabo la acción. Por eso, este enfoque es compatible con el constructivismo y le provee bases mucho más sólidas sobre las cuales discriminar aquellos acuerdos racionales y marcos de cooperación sobre los que puede hacerse inteligible y justificable una acción en una circunstancia social adecuada. ${ }^{11}$

En lo que sigue, se concretarán tres herramientas para el investigador social que permiten la pretensión introductoria de este artículo, a saber, con dichas herramientas debe disolverse la pregunta por la manera de investigar a la sociedad desde adentro a partir de un marco investigativo y científico adecuado.

11 Como ejemplo paradigmático, esta estructura es perfectamente compatible con la atribución de culpabilidades penales en la consecución de un acto particular. 


\section{La fuerza de las creencias}

La primera de las herramientas requiere complementar el fundamento racional con la noción de creencia. La razón para ello es que la idea de valor social que transversaliza las discusiones de la ciencia social, desde Weber (2012), o bien para estar de acuerdo con ella o para desecharla, se disuelve una vez atendido el uso del concepto de creencia. Esto es en caso en la medida en que una institución, entendida como un sistema normativo, tiene sentido en la aceptación generalizada de los individuos que instancian tales normas. Cuando se centra la mirada en el por qué de esta instanciación, se arriba frecuentemente a la noción de valor: el valor social que tiene esta institución. De ahí que sean frecuentes alusiones al valor de la tolerancia, el valor de la democracia, etc. La noción de creencia disuelve la discusión en la medida en que hace innecesaria la idea de valor dado que la creencia se articula directamente con la estructura racional de la institución.

Para comenzar, la definición más simple de creencia es una afirmación que una persona hace y sobre la que debe estar dispuesto a dar razones. Así, que alguien afirme en una entrevista de campo, da igual si es estructurada o no, "el problema es que en este país siempre se roban los recursos públicos", implica que la persona cree que "en $x$ país alguien se roba siempre los recursos públicos". Siguiendo la definición simple de creencia, puede preguntársele a la persona las razones que tiene para mantener dicha creencia. Para que sea una creencia válida, debe cumplir con los criterios de razonabilidad antedichos. Si fracasa en el cumplimiento, esto quiere decir que la creencia es incompleta, es decir, es una razón obstinada o una razón que escapa a las condiciones institucionales básicas. En este sentido, puede decirse que tal persona o no pertenece a la institución o no tiene un conocimiento o competencia adecuado en el funcionamiento institucional.

La anterior descripción de la pertenencia institucional es particularmente relevante porque hace explícitas las evidencias de una persona para mantener una creencia particular, así como de su rol y capacidad para jugar el juego institucional de interés. Si se extiende el ejemplo anterior, puede decirse que la obstinación en la creencia de la malversación de recursos inhabilita a la persona para una participación adecuada en los procesos de gobernanza: se pierde la legitimidad del proceso en la medida en que dicha persona, que debería ser competente en el juego de lo público, no juegue de manera adecuada.

El análisis institucional resultante requiere la discriminación de los roles de los actores a partir de las creencias y su grado de racionalidad en términos del sistema normativo que constituye la institución social. Es a partir de dichas creencias 
explícitas que la institucionalización es posible en términos de seguimiento de reglas. Corregir creencias obstinadas es una de las tareas más importantes del análisis institucional, pero también es una de las más olvidadas. Nuestra propia concepción obstinada de la importancia de la democracia (de su valor) nos ha hecho olvidar que las razones (mayoritariamente prácticas para su defensa) dependen de nuestro propio marco racional (Williams, 2008).

La primera herramienta analítica orienta en la discriminación de las creencias que se mantienen en una comunidad permitiendo una adecuada caracterización de:

- Espacios de conflicto y reconciliación. ¿Cuáles son las creencias de los actores intervinientes en el conflicto entre sí? El marco de creencias definido permite determinar la cosmovisión que perpetúa la situación del conflicto, tal y como se propone desde las metodologías más específicas en resolución de conflicto (Deutsch et al., 2005).

- Análisis de percepción, grupos de representación y construcción de discursos interculturales. La identidad de colectivos y grupos de representación dentro de una sociedad se identifican a partir de creencias. Existen diferentes metodologías que permiten hacer explícitas estas creencias: encuestas y entrevistas, administración colectiva del significado (Barnett, 2007), etc. El aporte de la discriminación de las creencias desde el enfoque de Lenguaje y acción es que permite establecer un marco de racionalidad contextualizado que estructure las diferentes expectativas y jerarquía de creencias de la instancia social analizada.

\section{Discurso e ideología}

En la investigación social, bajo el enfoque de Lenguaje y acción, no solo es posible esclarecer la institucionalización social por medio de las creencias. El marco racional que da pie a este análisis estructural también permite una discriminación práctica de las instancias discursivas que dinamizan las creencias. Como afirma Thompson (1987), "los componentes cognitivos (ideas, significados, actitudes, etc.) no fluyen sin rumbo por el mundo social, sino que circulan por él en forma de frases, expresiones, palabras, tanto en forma hablada como en forma escrita" (p. 517). El flujo de estos componentes de manera general desde dos aproximaciones: un análisis ideológico y un análisis teleofuncional.

La primera aproximación retoma la ya constituida metodología del análisis del discurso. En especial, el aspecto ideológico constriñe el análisis crítico del discur- 
so. Desde este enfoque, se busca identificar críticamente las relaciones asimétricas de poder y autoridad que se dan en las expresiones textuales sociopolíticas (van Dijk, 1993). De ahí que lo que llamamos análisis del discurso, por ejemplo, hace referencia a una "disciplina" o saber cuyo objeto de estudio es el discurso, esto es, los diferentes usos del lenguaje que hacen los hablantes en unas situaciones determinadas: política, literatura, comunicación, religión, entre otras. En este sentido, el análisis del discurso se fundamenta en la teoría de los actos de habla (Searle, 1969). Pero no es simplemente una caracterización deontológica de la acción: es la construcción de la realidad social a partir de las prácticas de institucionalización realizadas a través del lenguaje y la escritura.

Nótese la siguiente afirmación de Searle (2010): "Porque el poder político es un asunto de funciones de estatus, está en gran medida constituido lingüísticamente" (p. 169). En esta afirmación, está contenido el sentido del análisis del discurso, por lo que es importante analizarla brevemente. 1) Se afirma que el poder político es un asunto de funciones de estatus. Esto se debe a que, cuando alguien ejerce poder político propiamente hablando, está aludiendo a una institución y, por tanto, a las funciones estatus (como reglas constitutivas) que rigen dicha institución. 2) En la medida en que esas funciones están condicionadas lingüísticamente a partir de la estructura normativa que hace explícita la regla (supra), el poder político depende y se expresa a través de las prácticas lingüísticas. Esta es la conclusión más significativa de esta aproximación a la herramienta discursiva: que las prácticas textuales (entendiendo un texto como una expresión lingüística particular) suelen implicar un poder deontológico social y políticamente relevante de ser analizado.

La precisión importante que se hace del análisis del discurso a partir de Lenguaje y acción en el sentido previamente desarrollado es la definición de ideología. En vez de definirse a partir del estructuralismo, la ideología se entiende como el sistema de creencias (obstinadas) arraigado en un sociedad o comunidad particular que genera interacciones conflictivas. En esta medida, ideología no correspondería a un aspecto meramente estructural, sino que sería el proceso de un seguimiento de reglas racional (para tal comunidad) en detrimento de aquellos otros miembros perjudicados por estas creencias obstinadas. Este enfoque aporta una nueva luz sobre problemas como el racismo o la polarización política en la medida en que es racional para ciertos miembros (elitistas y poderosos) sostener tales creencias, mientras que las reglas institucionales completas se ven entorpecidas por la asimetría en estas relaciones de poder.

Por eso, es a partir de este concepto de ideología que se unen las dos aproximaciones. A saber, la segunda aproximación se define, de manera un poco vaga, 
como el análisis teleofuncional de la argumentación. Este enfoque atiende al uso ordinario del lenguaje y su estructuración lógica en la elaboración de argumentos a favor o en contra de diferentes posturas (Toulmin, 2007). Este enfoque, particularmente relevante para la comunicación y la retórica permite la descripción de los entramados inferenciales que dinamizan las creencias de una comunidad particular.

Piénsese en la argumentación que una comunidad particular pueda hacer para esclarecer la asignación del presupuesto participativo. La aproximación teleofuncional discriminaría las premisas con las cuales un grupo representativo defendería una asignación referida a la educación y no a eventos culturales. Dichas premisas pueden articularse con herramientas retóricas que apelen a la visión general del barrio, de su cohesión, del estado de cosas de la ciudad, etc. El resultado sería una argumentación completa en que sus componentes describen diferentes aspectos de las razones de una comunidad que asume como vinculantes en la justificación de sus decisiones internas.

La utilización metodológica de esta herramienta desde sus dos aproximaciones se concreta en las siguientes instancias:

- Comunicación política. La textualidad de los discursos referidos al poder, a la autoridad, a la tolerancia, etc., pueden discriminar procesos importantes sobre campañas políticas, alocuciones institucionales y polarización ciudadana.

- Lógica informal. En especial, la variante teleofuncional puede emplearse en análisis de lógica informal. El alcance de estos análisis comprende (de manera no exhaustiva) estudios de emociones discursivas, de polarización y discursos intolerantes, estructuración de instancias de deliberación pública, etc.

\section{Lenguaje y comportamiento racional}

Asimismo, el marco de creencias y su articulación a partir de razones puede explicar el comportamiento de otra manera muy particular, harto relevante para Lenguaje y acción como enfoque investigativo. Si la caja de herramientas se usa no para discriminar los contextos normativos amplios ni analizar instancias prácticas de preferencias discursivas, cabe la posibilidad de realizar una generalización abstracta de una pequeña parte de la práctica social: de un juego. La noción de juego como descriptor de las interacciones personales implica una modelación del comportamiento análoga a la relación entre teoría científica y 'realidad'. De lo 
que se trata no es de argumentar que la teoría sea la realidad, sino que la explica en un marco definido. Análogamente, un juego modela la interacción entre dos individuos racionales en la medida en que explica qué deberían hacer para contar como jugadores competentes de dicho juego.

Esta noción parte, evidentemente, de la noción pragmatista desarrollada más arriba. Si se quiere, puede verse como una metodologización de ella. Esta afirmación, un poco controversial, es importante en varios sentidos: primero, la teoría de las preferencias reveladas supone el seguimiento básico de reglas (Binmore, 2005, 2009; Elster, 2015). Cuando la teoría de juegos señala que no explica el comportamiento, sino que se limita a describirlo, implica, en un sentido muy importante, una aceptación de que en el principio era la acción. Segundo, la ambigüedad y tensión que existe entre estrategia y creencia, la cual solo se esclarece al optar por un modelo de análisis específico, resalta las complejas asunciones que se deben realizar al darle sentido a la acción racional. En vez de describir dos cosas prácticamente diferentes, estrategia y creencia definen dos apuestas particulares: por la teoría de juegos y por la teoría de la decisión. Más allá de adentrarnos en la discusión sobre la prioridad de una sobre la otra, para lo cual se remiten los argumentos desarrollados por Binmore (2009) y Spohn (1982), interesa resaltar que lo que suponen tales concepciones es una noción básica de adiestramiento (Binmore, 2009, p. 133): nos comportamos según como vemos las cosas (Schick, 2003, p. 5), creemos que se dan (Spohn, 2012) o como si actuáramos racionalmente (Binmore, 2009). En general, actuamos optimizando nuestras creencias, deseos y motivaciones (Elster, 2015).

Por esta razón, "una acción es racional si cumple tres criterios de optimalidad: la acción debe ser óptima dadas las creencias, las creencias deben estar tan bien fundadas como sea posible por la evidencia y la evidencia debe resultar de una inversión óptima en la recolección de información" (Elster, 2015, p. 235). Esta herramienta investigativa retoma, entonces, el desarrollo de las creencias que se hizo más arriba y lo contrasta con la evidencia epistémica soportada en información fiablemente recolectada. De manera más detallada: lo que un actor social percibe de un entorno o contexto de juego entra a actualizar o condicionar sus creencias de manera que selecciona las decisiones más estratégicas que lo llevarán a conseguir un objetivo específico.

Como ejemplo, piénsese en las interacciones de un actor en un país o municipio con baja estatalidad y con baja confianza en la fuerza pública (creencia inicial comunitariamente evidenciada). El actor recolecta información relevante cuando ve una instancia de violencia intrafamiliar que denuncia a la fuerza pública, pero 
esta es ineficaz en resolver la cuestión. Por otro lado, un grupo armado organizado es puesto al tanto y resuelve el problema de manera eficaz. La actualización o condicionalización de la creencia inicial según esta nueva evidencia es que para solucionar un conflicto intrafamiliar es más racional reportarlo al grupo armado.

Actuar de esta manera implica pertenecer a una comunidad, en la medida en que solo a partir de ciertas convenciones, normas sociales y expectativas institucionales mantenidas por una comunidad específica es posible predecir lo que desde esta teoría se denominan equilibrios en las interacciones. Aún más, solo es posible discriminar esta participación por la práctica básica de dar y pedir razones. La expresividad del comportamiento cotidiano solo puede ser alcanzada al racionalizar el comportamiento a partir de las razones que se puedan dar y pedir por este.

Nótese que el ejemplo no discrimina una base deontológica o legal en que se evite esta actualización por ser ilegal o moralmente mala. Un agente racional no siempre actúa según normas morales y normas jurídicas. Pero dichas normas sí son las que permiten interacciones mucho más estables en el juego de lo público. Por tanto, la tarea del investigador social que use esta herramienta será discriminar los incentivos relevantes en un marco de un aseguramiento de estado de cosas básico de juego según normas morales y jurídicas. Es decir, debe establecer cómo puede compatibilizarse el comportamiento efectivo de la comunidad con el cumplimiento de las normas sociales deseadas. Es, en cierto sentido, una inversión de la estructuración republicana de Pettit (supra).

Lo anterior no quiere decir que se reduzca la teoría a un campo puramente descriptivo y deje de lado lo normativo, sino que es el lenguaje el que permite desplegar la caja de herramientas de la que se vale la teorización completa de las interacciones humanas desde el punto de vista de los juegos o la toma de decisiones racionales.

\section{Conclusiones}

La investigación desde el enfoque de Lenguaje y acción parte, como se dijo desde el principio, del esclarecimiento de las preguntas que determinan el objetivo del científico social. A saber, el comportamiento humano está en el centro de la cuestión; de otro modo, no se situaría la pregunta en el ámbito de lo social. A partir de la concepción pragmática del lenguaje, se presentó una visión que hace inseparable la sociabilidad humana de las expresiones lingüísticas que las soportan. 
En este sentido, la investigación se condicionó a las herramientas que permiten desarrollar y explotar esta visión. Desde este enfoque, el esclarecimiento de las preguntas de investigación debe pasar por un riguroso tamiz de claridad lingüística. De la misma manera que en el discurso cotidiano distinguimos de manera comunitaria las preguntas que tiene o no sentido hacer sobre la realidad, en la investigación científica se tendrá que discriminar, dentro de cada comunidad académica y científica específica, qué preguntas tiene sentido o no hacer:

Preguntas como '¡esto es un árbol?' o '¡existen los unicornios?' se mueven bajo el mismo espíritu que centra la atención en Sobre la certeza, a saber: la duda de la existencia de mis manos. Podríamos decir que [...] no solo cabría responderle '¿qué quieres decir con esa pregunta?', sino 'no te entiendo, ¿aacaso estás confundido?’. (Santamaría, 2016, p. 212)

Parafraseando un poco, puede decirse que preguntas como '¿por qué la institucionalidad no tiene presencia en un territorio?' o ‘‘cómo es que podemos lograr la deconstrucción a partir de la subjetividad del individuo consciente de su clase?' no pueden tomarse en serio y habrá que responder a ellas con un '¡acaso estás confundido?'. Este enfoque se articula directamente a las conductas que, como advierte Winch (1990) desde Wittgenstein, son racionales en la medida en que se encuentran inmersas en prácticas sociales, en juegos de lenguaje que van configurando las propias reglas. Reglas que mantienen un carácter abierto, flexible, en el sentido de que es la dinámica del seguimiento de reglas el que constituye la verdadera naturaleza del comportamiento humano: "solo en la aplicación de un precedente anterior tiene que ser aplicado a un caso nuevo, que la naturaleza de la regla se vuelve aparente" (Winch, 1990, p. 62).

Por eso, la investigación social es un asunto de dinámica: las reglas vivas en el comportamiento pueden hacerse explícitas y normalmente lo hacen en circunstancias básicas. Pero no solo se trata de reconocer su explicitud, sino de entender su dinámica. Las tres herramientas desarrolladas muestran las diferentes alternativas para hacer explícitas no solo las reglas, sino también la dinámica social que las instancia, esto es, que las institucionaliza. Por eso, la importancia de partir del marco de creencias y las razones. Son ellas las que dotan de sentido el enfoque en la medida en que a partir de estas se basa toda práctica de razonabilidad humana (lo que sería en sí mismo un pleonasmo). 


\section{Referencias}

Acero, J. J. (2007). Filosofía del lenguaje I. Semántica. Trotta.

Barnett, W. (2007). Making Social Worlds. A Communication Perspective. Blackwell.

Binmore, K. (2005). Natural Justice. Oxford University Press.

Binmore, K. (2009). Rational Decisions. Oxford University Press.

Brandom, R. (2005). Hacerlo explícito: razonamiento, representación y compromiso discursivo. Herder.

Brandom, R. (2008). Between Saying and Doing: Towards an Analytic Pragmatism. Oxford University Press.

Davidson, D. (2001). Essays on Actions and Events. Oxford University Press

Deutsch, M., Coleman, P. y Marcus, E. (2005). The Handbook of Conflict Resolution. Theory and Practice. Josey-Bass.

Elster, J. (2015). Explaining Social Behavior: More Nuts and Bolts for the Social Sciences. Cambridge University Press.

Fann, K. T. (2015). Wittgenstein's Conception of Philosophy. Patridge.

Korta, K. y Perry, J. (2020). Pragmatics. En E. N. Salta (ed.), The Stanford Encyclopedia of Philosophy (Spring 2020 Edition). https://plato.stanford.edu/archives/spr2020/ entries/pragmatics/

Locke, J. (1999). Ensayo sobre el entendimiento humano. Fondo de Cultura Económica.

McDowell, J. (1998). Mind, Value and Reality. Harvard University Press.

Peirce, C. S. (2012). Cómo esclarecer nuestras ideas. En N. Houser y C. Kloesel (eds.), Obra filosófica reunida. Tomo I: 1867-1893, (pp. 172-188). Fondo de Cultura Económica.

Pettit, P. (1997). Republicanism. A Theory of Freedom and Government. Oxford University Press.

Rawls, J. (1955). Two Concepts of Rules. The Philosophical Review, 64(1), 3-32. https:// doi.org/10.2307/2218309

Rawls, J. (1996). Political Liberalism. Columbia University Press.

Rawls, J. (1999). A Theory of Justice. Revised Edition. The Belknap Press.

Rodríguez Ortiz, A. y Ramírez - Vallejo, D. A. (2021). Wittgenstein y Searle: entre los juegos de lenguaje y los actos de habla. En F. Santamaría Velasco, N. Rosanía Maza y K. Cárdenas Almanza (eds.), Perspectivas wittgensteinianas: lenguaje, significado y acción (pp. 403-446). Tirant lo Blanch.

Ruiz-Martínez, S. y Rosanía Maza, N. A. (2021). Tres coordenadas lógicas para la objetividad. En F. Santamaría Velasco, N. A. Rosanía Maza y K. Cárdenas Almanza, Perspectivas Wittgensteinianas: lenguaje, significado y acción (pp. 101-140). Tirant lo Blanc.

Santamaría Velasco, F. (2016). Hacer mundos: el nombrar y la significatividad. Siglo del Hombre.

Santamaría Velasco, F. y Ruiz-Martínez, S. (2019). ¿Qué significa pertenecer a una comunidad? Disputatio Philosophical Research Bulletin, 8(9), 1-27.

Sartori, G. (2002). La política: lógica y método en ciencias sociales. Fondo de Cultura Económica.

Searle, J. R. (1969). Speech Acts: An Essay in the Philosophy of Language. Cambridge University Press. 
108 | Freddy Santamaría Velasco, Simón Ruiz-Martínez Lenguaje y acción: creencias, instituciones y política

Searle, J. R. (1995). The Construction of Social Reality. The Free Press.

Searle, J. R. (2010). Making the Social World. The Structure of Human Civilization. Oxford University Press.

Sellars, W. (1997). Empiricism and the Philosophy of Mind. Harvard University Press.

Sellars, W. (2005). Pure Pragmatics and Possible Worlds. Ridgeview.

Shick, F. (2000). Hacer elecciones. Gedisa.

Shick, F. (2003). Ambiguity and Logic. Cambridge University Press.

Sosa, E. (2011). Knowing Full Well. Princeton University Press.

Spohn, W. (1982). How to Make Sense of Game Theory. En W. Stegmüller, W. Balzer y W. (eds.), Philosophy of Economics. Studies in Contemporary Economics (vol. 2, pp. 239-270). Springer.

Spohn, W. (2012). The Laws of Belief. Ranking Theory \& Its Philosophical Applications. Oxford University Press.

Thompson, J. B. (1987). Language and Ideology: A Framework for Analysis. The Sociological Review, 35(3), 516-536. https://doi.org/10.1111/j.1467-954X.1987.tb00554.x

Tomasini Bassols, A. (2016). Reasons and causes: Wittgenstein versus Davidson. Praxis Filosófica, 43, 13-36.

Toulmin, S. (2007). Los usos de la argumentación. Península.

Van Dijk, T. A. (1993). Principles of Critical Discourse Analysis. Discourse \& Society, 4(2), 249-283. https://doi.org/10.1177/0957926593004002006

Waismann, F. (1968). How I See Philosophy. Macmillan.

Weber, M. (2012). "Objectivity" and Knowledge in Social Science and Social Policy. Routledge.

Williams, B. (2008). In the Beginning was the Deed: Realism and Moralism in Political Argument. Princeton University Press.

Winch, P. (1990). The Idea of a Social Science and Its Relation to Philosophy. Routledge.

Wittgenstein, L. (1969). On Certainty. Blackwell.

Wittgenstein, L. (2009). Philosophical Investigations (3. ${ }^{a}$ ed., P. M. S. Hacker y J. Schulte, eds.). Wiley-Blackwell. 